\title{
Polaronic trapping of electrons and holes by native defects in anatase $\mathrm{TiO}_{2}$
}

\author{
Benjamin J. Morgan* and Graeme W. Watson ${ }^{\dagger}$ \\ School of Chemistry, Trinity College, University of Dublin, Dublin 2, Ireland
}

(Received 18 November 2009; published 16 December 2009)

\begin{abstract}
We have investigated the formation of native defects in anatase $\mathrm{TiO}_{2}$ using density functional theory (DFT) modified with on-site Coulomb terms $(\mathrm{DFT}+U)$ applied to both Ti $d$ and $\mathrm{O} p$ states. Oxygen vacancies and titanium interstitials are found to be deep donors that trap two and four electrons, with transition levels that explain the two features seen in deep level transient spectroscopy experiments. Titanium vacancies are deep acceptors accommodating four holes. Self-trapping of both electrons and holes is also predicted. In all cases both donor and acceptor trap states correspond to strongly localized small polarons, in agreement with experimental EPR data. Variation in defect formation energies with stoichiometry explains the poor hole-trapping of reduced $\mathrm{TiO}_{2}$.
\end{abstract}

DOI: $10.1103 /$ PhysRevB.80.233102

PACS number(s): 71.38.Ht, 71.55.-i, 82.50. $-\mathrm{m}$

In semiconductors used in photocatalysis and photovoltaics, the primary process is the light-induced production of charge carriers. ${ }^{1}$ Photocatalysis proceeds by photoexcitation of electrons to unoccupied bands, producing free electronhole pairs. These charge carriers then typically undergo trapping, followed by diffusion to the surface where they can initiate reactions of adsorbed molecules. Electron-hole recombination is often a major competing process, and it is highly desirable to promote charge separation and inhibit subsequent charge-carrier-recombination in order to improve the quantum efficiency of photocatalytic processes.

$\mathrm{TiO}_{2}$ has been widely studied as a photocatalyst for the degradation of environmental pollutants, ${ }^{1}$ and for water splitting. ${ }^{2}$ Nanocrystalline anatase $\mathrm{TiO}_{2}$ is often used, since it is more photocatalytically active than the ground-state polymorph, rutile, and the diffusion pathways of photogenerated charge carriers to the surface are shortened, resulting in increased quantum efficiencies.

Following photoexcitation, trapping of charge carriers occurs on a nanosecond time scale. ${ }^{3}$ Defects are thought to play a critical role in the trapping process by acting as recombination centers. For example, $n$-type doping of anatase reduces the photocatalytic activity of experimental samples, ${ }^{4}$ and reduced anatase is less efficient than stoichiometric $\mathrm{TiO}_{2}$ in the trapping of photogenerated holes. ${ }^{5}$ Understanding the interaction of defects with charge carriers is essential for the optimization of $\mathrm{TiO}_{2}$ samples for photocatalysis. Electronand hole traps have both been observed in EPR spectra. ${ }^{5-8}$ These data have been interpreted as charge localization at single atoms to give small polarons: hole trapping has been associated with $\mathrm{O}^{-}$sites; $\mathrm{O}_{\mathrm{O}}^{\circ}$, and electron trapping with $\mathrm{Ti}^{3+}$ species; $\mathrm{Ti}_{\mathrm{Ti}}^{\prime}$. Additional evidence for charge localization at $\mathrm{Ti}^{3+}$ states in $n$-type $\mathrm{TiO}_{2}$ comes from core-level XPS shifts, and characteristic gap-state features observable in UPS spectra, with samples annealed to produce oxygen vacancies giving identical EPR signals to those seen following uv irradiation. Deep traps 0.9 and $0.5 \mathrm{eV}$ below the conduction band edge have also been identified with deep level transient spectroscopy (DLTS). ${ }^{9}$

Na-Phattalung et al. have previously reported local density approximation (LDA)-density functional theory (DFT) calculations that predicted that both the oxygen vacancy $\left(V_{\mathrm{O}}\right)$ and titanium interstitial $\left(\mathrm{Ti}_{\mathrm{i}}\right)$ are shallow donors, and the titanium vacancy $\left(V_{\mathrm{Ti}}\right)$ is a shallow acceptor, with associated defect states corresponding to metallic bands at the valence and conduction band edges. ${ }^{10}$ Osorio-Guillén et al. used generalized gradient approximation (GGA)-DFT with a posteriori band-gap corrections, and in contrast reported deep donor levels for $V_{\mathrm{O}}$ and $\mathrm{Ti}_{\mathrm{i}}$, with the associated defect states at the bottom of the conduction band. ${ }^{11}$ The prediction of delocalized defect states, however, is inconsistent with the localized polaronic description from experimental data. ${ }^{5-8}$

The failure of LDA and GGA DFT functionals to be unable to correctly describe defect states is well known for a number of wide-gap oxides. ${ }^{12-15}$ This is due to the selfinteraction error (SIE) inherent to such functionals, which results in an artificial bias toward delocalization of partially occupied states. ${ }^{16}$ This problem is acute for localized metal $d$ and $f$ states, and also exhibited for oxygen $p$ states. ${ }^{12,15}$ For $\mathrm{TiO}_{2}$ it has been shown that experimental features of excess electron states that accompany $n$-type doping are only reproduced when the self-interaction error is corrected for. ${ }^{17-22}$ The need for self-interaction corrections in describing the polaronic nature of excess electrons and holes in $\mathrm{TiO}_{2}$ has also been discussed by Deskins et al. ${ }^{23,24}$

In this Brief Report we report a DFT $+U$ examination of the transition levels and single particle levels of $n$ - and $p$-type native defects in anatase $\mathrm{TiO}_{2}$. In order to correctly describe these states we apply $+U$ corrections to both Ti $d$ and $\mathrm{O} p$ states. $\mathrm{DFT}+U\left\{\mathrm{Ti}_{d}\right\}$ has been demonstrated to be suitable for $n$-type defects in $\mathrm{TiO}_{2}$, yet no calculations to date have addressed the problem of the self-interaction error (SIE) for O-derived defect states. Using an $a b$ initio $U\left\{\mathrm{O}_{p}\right\}$ value that correctly places the unoccupied $\mathrm{O} 2 p$ states relative to the valence band edge, we find that: $V_{\mathrm{O}}$ and $\mathrm{Ti}_{\mathrm{i}}$ are deep donors, with the neutral defects producing single particle levels in the band gap; $V_{\mathrm{Ti}}$ is a quadruple deep donor, again with single particle levels in the band gap; both electrons and holes are predicted to undergo self-trapping; defect states for both native defects and self-trapped charge carriers correspond to holes or electrons trapped as small polarons to give $\mathrm{O}_{\mathrm{O}}^{\bullet}$ and $\mathrm{Ti}_{\mathrm{Ti}}^{\prime}$.

Method. To counteract the problems associated with the SIE inherent to standard density functionals we have used 


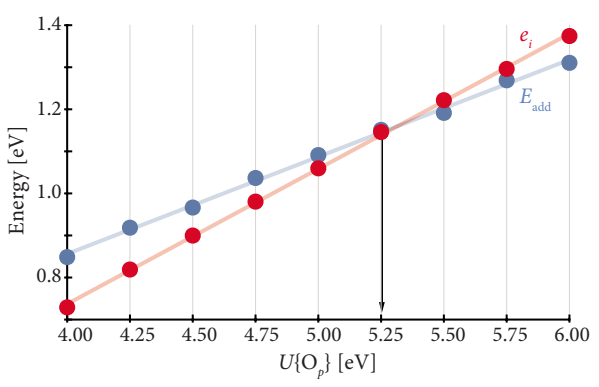

FIG. 1. (Color online) The electron addition energy, $E_{\text {add }}$ $=E_{N+1}-E_{N}$, and the hole state eigenvalue (relative to the VBM) of the +1 charge state of anatase $\mathrm{TiO}_{2}$. The arrow shows the value of $U\left(\mathrm{O}_{\mathrm{p}}\right)$ for which Eq. (1) is satisfied.

the Perdew-Burke-Ernzerhof GGA functional, ${ }^{25}$ supplemented with Dudarev on-site Hubbard corrections applied to both Ti $d$ and $\mathrm{O} p$ states; DFT $+U\left\{\mathrm{Ti}_{d}, \mathrm{O}_{p}\right\} .{ }^{26} U\left\{\mathrm{Ti}_{d}\right\}$ was 4.2 $\mathrm{eV}$, which gives the correct splitting between occupied and unoccupied Ti $d$ states for $\mathrm{O}$ vacancies at the rutile $\mathrm{TiO}_{2}$ (110) surface $^{18}$ and has been used to model both native $n$-type defects, and $\{\mathrm{Nb}, \mathrm{Ta}\}$-doping of anatase $\mathrm{TiO}_{2} \cdot{ }^{21,22} \mathrm{An}$ ab initio $U\left\{\mathrm{O}_{p}\right\}$ value of $5.25 \mathrm{eV}$ was used, as described below. Calculations were performed in VASP (Refs. 27 and 28) with a plane-wave basis cutoff of $500 \mathrm{eV}$. Structures were relaxed until the forces on all atoms were less than $0.01 \mathrm{eV} \AA^{-1}$. Interactions between core and valence electrons were described using the projector augmented-wave method. ${ }^{29}$ Calculations were performed using $3 \times 3 \times 1$ supercells (108 atoms) for all defects, except for the titanium vacancy, where $3 \times 3 \times 2$ supercells (216 atoms) were required to minimize interactions along the $c$ direction. $\Gamma$-centered $2 \times 2 \times 2 k$-point sampling was used for all calculations. All calculations were spin polarized.

To determine $U\left\{\mathrm{O}_{p}\right\}$ we used the $a b$ initio fitting procedure of Lany and Zunger. ${ }^{15}$ For the (unknown) exact density functional the change in energy of the system when part of an electron is added or removed is linear. ${ }^{16}$ For addition of an electron to a hole state, this condition requires that

$$
E(N+1)-E(N)=e_{i}(N) .
$$

Here $E(N+1)-E(N)$ is the electron addition energy, i.e., the difference in energy between the system with a self-trapped hole present (the +1 charge state), and the neutral system; where both are calculated at the optimized geometry for the hole system; and $e_{i}(N)$ is the eigenvalue of the hole state relative to the valence band maximum. Figure 1 shows the variation in $[E(N+1)-E(N)]$ and $e_{i}(N)$ for the +1 charge state of stoichiometric anatase $\mathrm{TiO}_{2}$. Equation (1) is satisfied for $U\left\{\mathrm{O}_{p}\right\}=5.25 \mathrm{eV}$, giving the correct splitting between occupied and unoccupied $\mathrm{O} 2 p$ states. The formation enthalpy of a defect with charge state $q$ is given by

$$
\Delta H_{\mathrm{f}}(D, q)=\left(E^{D, q}-E^{H}\right)+\sum_{i} n_{i}\left(E_{i}+\mu_{i}\right)+q E_{\mathrm{F}},
$$

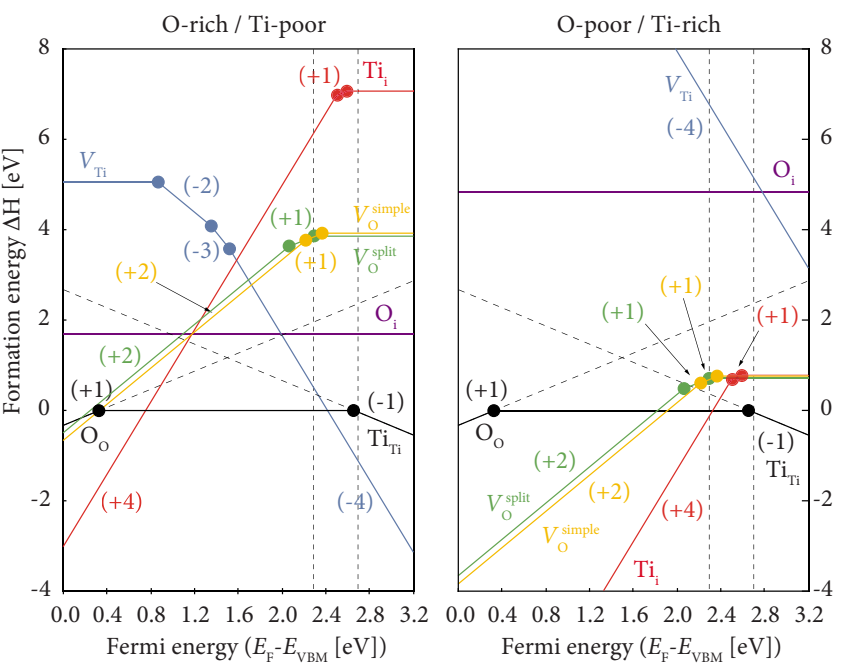

FIG. 2. (Color online) Formation energies for intrinsic defects in anatase $\mathrm{TiO}_{2}$. The solid dots denote transition levels $\epsilon\left(q / q^{\prime}\right)$. The diagonal dashed lines give the energies of metastable self-trapped electrons and holes produced by photoexcitation. The vertical dashed lines are the DLTS levels reported by Miyagi et al. ${ }^{9}$

$$
\text { where } E_{\mathrm{F}}=\Delta E_{\mathrm{F}}+\epsilon_{\mathrm{VBM}}^{H}+\Delta v(D) .
$$

$E^{H}$ is the total energy of the stoichiometric host supercell and $E^{D, q}$ is the total energy of the defective cell. Elemental reference energies, $E_{i}$, were obtained from calculation on the constituent elements in their standard states, i.e., $\mathrm{O}_{2(\mathrm{~g})}$ and $\operatorname{Ti}_{(\mathrm{s})} \cdot n_{\alpha}$ is the number of atoms transferred to or from reservoirs with respective chemical potentials $\mu_{\alpha}$. These chemical potentials represent specific equilibrium growth conditions, within the general constraint of the formation enthalpy of anatase $\mathrm{TiO}_{2}: \mu_{\mathrm{Ti}}+2 \mu_{\mathrm{O}}=\Delta H_{\mathrm{f}}^{\mathrm{TiO}_{2}}=-8.11 \mathrm{eV}$. The upper limit for $\mu(\mathrm{O})$, which characterizes an O-rich/Ti-poor environment, is governed by the formation of $\mathrm{O}_{2(\mathrm{~g})} ; \mu(\mathrm{O})$ $=0 \mathrm{eV}$ and $\mu(\mathrm{Ti})=-8.11 \mathrm{eV}$. The O-poor/Ti-rich lower limit to $\mu(\mathrm{O})$ is constrained by the formation of $\mathrm{Ti}_{2} \mathrm{O}_{3}$; $2 \mu(\mathrm{Ti})+3 \mu(\mathrm{O}) \leq \Delta H_{\mathrm{f}}^{\mathrm{Ti}_{2} \mathrm{O}_{3}}=-13.08 \mathrm{eV}$, which gives $\mu(\mathrm{O})$ $=-3.14 \mathrm{eV} ; \mu(\mathrm{Ti})=-1.82 \mathrm{eV} . \Delta E_{\mathrm{F}}$ can range from the $\operatorname{VBM}\left(E_{\mathrm{F}}=0 \mathrm{eV}\right)$ to the CBM $\left(E_{\mathrm{F}}=3.2 \mathrm{eV}\right) . \epsilon_{\mathrm{VBM}}^{H}$ is the energy of the VBM in the stoichiometric system, and $\Delta v(D)$ is a core-level alignment between the stoichiometric and defect cells, obtained by taking the difference of $\mathrm{O} 1 \mathrm{~s}$ corelevel energies for an oxygen atom well removed from the defect position. Thermodynamic ionization (transition) levels, $\epsilon_{D}\left(q / q^{\prime}\right)$, are given by the Fermi energy at which the energies of two charge states, $q$ and $q^{\prime}$, of the defect $D$ are equal;

$$
\epsilon_{D}\left(q / q^{\prime}\right)=\frac{\Delta H_{\mathrm{f}}(D, q)-\Delta H_{\mathrm{f}}\left(D, q^{\prime}\right)}{q^{\prime}-q} .
$$

Results. Fig. 2 shows calculated formation energies and transition levels for $\mathrm{Ti}_{\mathrm{Ti}}, V_{\mathrm{O}}, \mathrm{O}_{\mathrm{i}}$, and $V_{\mathrm{Ti}}$. Oxygen vacancy data are presented for both the "simple" $V_{\mathrm{O}}$; where the excess electrons are localized at the two $\mathrm{Ti}$ sites neighboring the vacancy; and for the "split" $V_{\mathrm{O}}$; where one electron is localized at a next-nearest Ti site. ${ }^{21}$ Also shown are the calculated 
TABLE I. Single particle levels relative to the valence band maximum in eV. For the defects with occupied levels, the conduction band offset at the $\Gamma$ point is given in brackets.

\begin{tabular}{|c|c|c|c|c|c|c|c|c|c|c|c|c|c|}
\hline \multicolumn{2}{|c|}{$V_{\mathrm{O}}^{\text {simple }}$} & \multicolumn{2}{|c|}{$V_{\mathrm{O}}^{\text {split }}$} & \multicolumn{4}{|c|}{$\mathrm{Ti}_{\mathrm{i}}$} & \multicolumn{4}{|c|}{$V_{\mathrm{Ti}}$} & \multirow{2}{*}{$\frac{\mathrm{Ti}_{\mathrm{Ti}}}{-1}$} & \multirow{2}{*}{$\frac{\mathrm{O}_{\mathrm{O}}}{+1}$} \\
\hline 0 & +1 & 0 & +1 & 0 & +1 & +2 & +3 & 0 & -1 & -2 & -3 & & \\
\hline & & & & $1.88(0.74)$ & & & & 1.91 & & & & & \\
\hline & & & & $1.64(0.98)$ & $1.62(1.00)$ & & & 1.76 & 1.86 & & & & \\
\hline $1.47(1.15)$ & & $1.35(1.27)$ & & $1.32(1.30)$ & $1.45(1.17)$ & $1.53(1.09)$ & & 1.76 & 1.60 & 1.90 & & & \\
\hline $1.14(1.48)$ & $1.27(1.35)$ & $1.26(1.36)$ & $1.25(1.37)$ & $0.88(1.74)$ & $0.88(1.74)$ & 0.88 & 0.91 & 1.53 & 1.48 & 1.60 & 1.64 & 1.80 & 1.09 \\
\hline
\end{tabular}

energies for self-trapped holes, $\mathrm{O}_{\mathrm{O}}^{\cdot}$ and electrons, $\mathrm{Ti}_{\mathrm{Ti}}^{\prime} \cdot \mathrm{Ti}_{\mathrm{i}}$ and $V_{\mathrm{O}}$ have deep transition levels, as predicted by OsorioGuillén et al. ${ }^{11}$ The single particle levels associated with the neutral and intermediate-charge defects are in the band gap; Table I and Fig. 3; in contrast to previous calculations. ${ }^{10,11}$ These deep states correspond to electrons trapped at Ti sites as small polarons. For the neutral $V_{\mathrm{O}}$ two electrons are close to the vacancy site, whereas for $\mathrm{Ti}_{\mathrm{i}}$ one electron associated with the deepest level $\sim 1.72 \mathrm{eV}$ below the CBM is trapped at the interstitial $\mathrm{Ti}$ site, and the other three are localized at lattice $\mathrm{Ti}$ sites neighboring to the interstitial, giving $\left[\mathrm{Ti}_{\mathrm{i}}^{\bullet \bullet \bullet}+3 \mathrm{Ti}_{\mathrm{Ti}}^{\prime}\right]^{21}$

The transition levels for $V_{\mathrm{Ti}}$ are also within the band gap. The single particle levels for these hole states are well separated from the VBM, and correspond to small polarons at up to four of the six $\mathrm{O}$ sites surrounding the vacancy; Fig. 4. Such trapping of holes at individual defect site ligands is well known for a range of wide-gap oxides, ${ }^{30,31}$ and polaronic binding of holes has been predicted for Al-doped $\mathrm{TiO}_{2}{ }^{32}$ For the neutral vacancy the four holes are localized at two equatorial and two apical sites of the distorted octahedron around the vacancy site. As the Fermi level is increased, the apical sites are detrapped first, producing well separated $\epsilon(0 /-2)$ and $\{\epsilon(-2 /-3), \epsilon(-3 /-4)\}$ transition levels. $\mathrm{O}_{i}$ is present as a peroxide ion at an oxygen lattice site, giving $\left(\mathrm{O}_{2}\right)_{\mathrm{O}}^{\times}$, as described by Na-Phattalung et al. ${ }^{10}$ Since this defect neither donates nor accepts charge carriers its

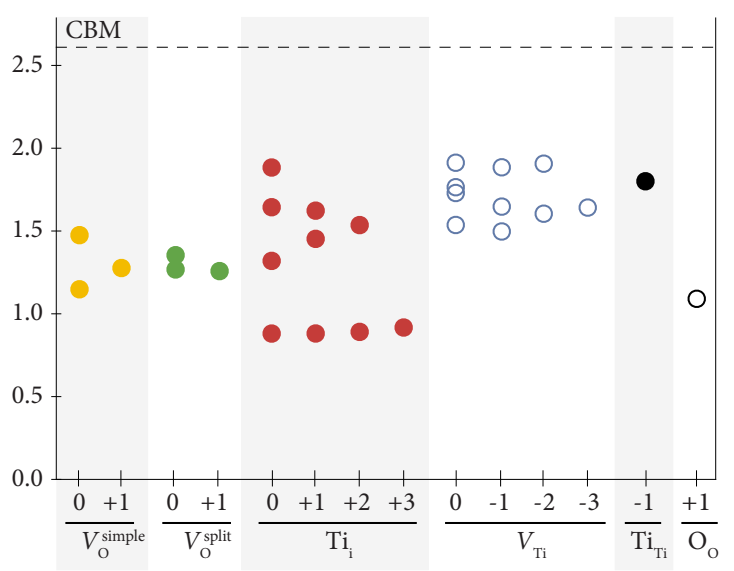

FIG. 3. (Color online) Single particle levels for all defect states showing levels in the band gap. Filled circles are occupied levels due to $n$-type defects, and empty circles are unoccupied levels due to $p$-type defects. presence is expected to have little effect on the photochemistry of the material.

Both electrons and holes are also predicted to undergo self-trapping. Self-trapped electrons correspond to strongly localized polaronic states at single $\mathrm{Ti}$ centers; $\mathrm{Ti}_{\mathrm{Ti}}^{\prime}$; and selftrapped holes are similarly strongly localized at single $\mathrm{O}$ centers; $\mathrm{O}_{\mathrm{O}}^{\cdot}$. The energy of a self-trapped photogenerated electron-hole pair is $2.35 \mathrm{eV}{ }^{33}$ The localized nature of the defect states is in agreement with the experimental EPR data, ${ }^{5}$ in contrast to previous LDA/GGA studies. ${ }^{10,11}$

Miyagi et al. have reported DLTS data for anatase $\mathrm{TiO}_{2}$ thin films showing deep trap levels 0.5 and $0.9 \mathrm{eV}$ below the conduction band edge, with the trap at $0.9 \mathrm{eV}$ present at a greater concentration. ${ }^{9}$ Our calculated $\epsilon(0 /+1)$ transition levels for $\mathrm{Ti}_{\mathrm{Ti}}$ and $V_{\mathrm{O}}^{\text {simple }}$ are 0.60 and $0.91 \mathrm{eV}$ from the experimental CBM of $3.2 \mathrm{eV}$, leading us to assign the trap at $0.5 \mathrm{eV}$ to $\mathrm{Ti}_{\mathrm{Ti}}$, and the trap at $0.9 \mathrm{eV}$ to $V_{\mathrm{O}}$; Fig. 2 .

The calculated defect formation energies for O-rich/Tipoor conditions are consistent with the known formation of charge-compensating $V_{\mathrm{Ti}}$ when $\mathrm{TiO}_{2}$ is doped $n$-type (with, e.g., $\mathrm{Nb}$ ) under ambient oxygen partial pressures. ${ }^{34,35}$ The variation in defect formation energies with sample composition also explains the reported absence of an EPR signal for photogenerated holes in reduced $\mathrm{TiO}_{2} .{ }^{5}$ Under O-poor/Tirich conditions oxygen vacancies and titanium interstitials form easily, whereas the formation energy of compensating $V_{\mathrm{Ti}}$ is very high. Hence the Fermi level is high in the band gap, and neutral oxygen vacancies and titanium interstitials, or even self-trapped electrons, will be present at high concentrations, and will act as recombination centers.

Summary. We have used DFT $+U$ with on-site Coulomb

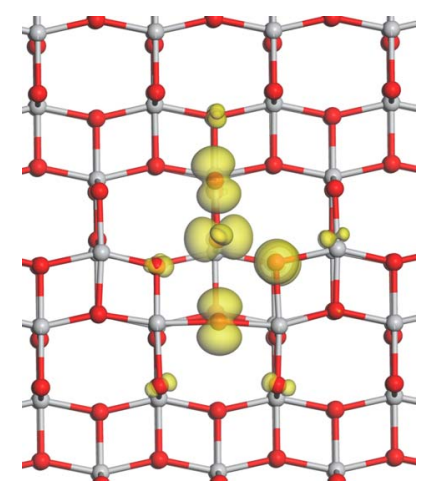

FIG. 4. (Color online) Spin density for the Ti vacancy defect state in anatase $\mathrm{TiO}_{2}$. The charge isosurface is shown at 0.05 e $\AA^{-3}$. 
corrections for both $\mathrm{Ti} d$ and $\mathrm{O} p$ states to study the native defects of anatase $\mathrm{TiO}_{2}$. This corrects for the self-interaction error of $n$ - and $p$-type defect states, and predicts that $V_{\mathrm{O}}, \mathrm{Ti}_{\mathrm{Ti}}$, and $V_{\mathrm{Ti}}$ are characterized by strongly localized electrons or holes, in agreement with experimental EPR data. We find that transition levels for these defects are deep, and assign the DLTS signals at 0.9 and $0.5 \mathrm{eV}$ to $V_{\mathrm{O}}$ and $\mathrm{Ti}_{\mathrm{Ti}}$, respectively. Calculated formation energies allow the experimentally observed variation in photo-hole lifetimes with stoichi- ometry to be understood in terms of the number of "holekiller" $n$-type electron traps present.

This research was supported by Science Foundation Ireland under Grant No. 06/IN/1/I92, and supplement 06/IN/1/ I92/EC07. Calculations were performed on the IITAC supercomputer as maintained by the Trinity Centre for High Performance Computing (TCHPC), and the Stokes supercomputer; maintained by the Irish Centre for High-End Computing (ICHEC). *morganb@tcd.ie

†watsong@tcd.ie

${ }^{1}$ A. L. Linsebigler, G. Lu, and J. T. Yates, Jr., Chem. Rev. 95, 735 (1995).

${ }^{2}$ A. Fujishima and K. Honda, Nature 238, 37 (1972).

${ }^{3}$ D. P. Colombo and R. M. Bowman, J. Phys. Chem. 99, 11752 (1995).

${ }^{4}$ T. Miyagi, M. Kamei, I. Sakaguchi, T. Misuhashi, and A. Yamzaki, Jpn. J. Appl. Phys. 43, 775 (2004).

${ }^{5}$ T. Berger, M. Sterrer, O. Diwald, E. Knözinger, D. Panayotov, T. L. Thompson, and J. T. Yates, Jr., J. Phys. Chem. B 109, 6061 (2005).

${ }^{6}$ R. F. Howe and M. Grätzel, J. Phys. Chem. 91, 3906 (1987).

${ }^{7}$ S. Zhou, A. Čižmar, K. Potzger, M. Krause, G. Talut, M. Helm, J. Fassbender, S. A. Zvyagin, J. Wosnitza, and H. Schmidt, Phys. Rev. B 79, 113201 (2009).

${ }^{8}$ S. Yang, L. E. Halliburton, A. Manivannan, P. H. Bunton, D. B. Baker, M. Klemm, S. Horn, and A. Fujishima, Appl. Phys. Lett. 94, 162114 (2009).

${ }^{9}$ T. Miyagi, M. Kamei, T. Mitsuhashi, and A. Yamazaki, Appl. Phys. Lett. 83, 1782 (2003).

${ }^{10}$ S. Na-Phattalung, M. F. Smith, K. Kim, M.-H. Du, S.-H. Wei, S. B. Zhang, and S. Limpijumnong, Phys. Rev. B 73, 125205 (2006).

${ }^{11}$ J. Osorio-Guillén, S. Lany, and A. Zunger, Phys. Rev. Lett. 100, 036601 (2008).

${ }^{12}$ M. Nolan and G. W. Watson, J. Chem. Phys. 125, 144701 (2006).

${ }^{13}$ D. O. Scanlon, A. Walsh, B. J. Morgan, M. Nolan, J. Fearon, and G. W. Watson, J. Phys. Chem. C 111, 7971 (2007).

${ }^{14}$ D. O. Scanlon, B. J. Morgan, G. W. Watson, and A. Walsh, Phys. Rev. Lett. 103, 096405 (2009).

${ }^{15}$ S. Lany and A. Zunger, Phys. Rev. B 80, 085202 (2009).

${ }^{16}$ P. Mori-Sánchez, A. J. Cohen, and W. Yang, Phys. Rev. Lett. 100, 146401 (2008).

${ }^{17}$ C. Di Valentin, G. Pacchioni, and A. Selloni, Phys. Rev. Lett.
97, 166803 (2006).

${ }^{18}$ B. J. Morgan and G. W. Watson, Surf. Sci. 601, 5034 (2007).

${ }^{19}$ C. J. Calzado, N. C. Hernández, and J. F. Sanz, Phys. Rev. B 77, 045118 (2008)

${ }^{20}$ C. Di Valentin, G. Pacchioni, and A. Selloni, J. Phys. Chem. C 113, 20543 (2009).

${ }^{21}$ B. J. Morgan and G. W. Watson, J. Phys. Chem. C (to be published).

${ }^{22}$ B. J. Morgan, D. O. Scanlon, and G. W. Watson, J. Mater. Chem. 19, 5175 (2009).

${ }^{23}$ N. A. Deskins and M. Dupuis, Phys. Rev. B 75, 195212 (2007).

${ }^{24}$ N. A. Deskins and M. Dupuis, J. Phys. Chem. C 113, 346 (2009).

${ }^{25}$ J. P. Perdew, K. Burke, and M. Ernzerhof, Phys. Rev. Lett. 77, 3865 (1996).

${ }^{26}$ S. L. Dudarev, G. A. Botton, S. Y. Savrasov, C. J. Humphreys, and A. P. Sutton, Phys. Rev. B 57, 1505 (1998).

${ }^{27}$ G. Kresse and J. Hafner, Phys. Rev. B 49, 14251 (1994).

${ }^{28}$ G. Kresse and J. Furthmuller, Comput. Mater. Sci. 6, 15 (1996).

${ }^{29}$ P. E. Blöchl, Phys. Rev. B 50, 17953 (1994).

${ }^{30}$ O. F. Schirmer, J. Phys.: Condens. Matter 18, R667 (2006).

${ }^{31}$ A. M. Stoneham, J. Gavartin, A. L. Shluger, A. V. Kimmel, D. M. Ramo, H. M. Ronnow, G. Aeppli, and C. Renner, J. Phys.: Condens. Matter 19, 255208 (2007).

${ }^{32}$ A. Stashans and S. Bermeo, Chem. Phys. 363, 100 (2009).

${ }^{33}$ In general, the self-energy of small polarons includes contributions due to lattice fluctuations that can be expressed as nonadiabatic tunneling terms [J. T. Devreese and A. S. Alexandrov, Rep. Prog. Phys. 72, 066501 (2009)]. Since the defect structures correspond to optimization at $0 \mathrm{~K}$, any vibrational motion is "frozen out" and the self-energies presented here correspond to the adiabatic limit.

${ }^{34}$ J. F. Baumard and E. Tani, J. Chem. Phys. 67, 857 (1977).

${ }^{35}$ S. Zhang, S. B. Ogale, W. Yu, X. Gao, T. Liu, S. Ghosh, G. P. Das, A. T. S. Wee, R. L. Greene, and T. Venkatesan, Adv. Mater. 21, 2282 (2009). 